\title{
SECOND HARMONIC RESONANCE IN MAGNETOHYDRODYNAMIC JET
}

\author{
H.K. KHOSLA ${ }^{1}$ and R.K. CHHABRA ${ }^{2}$
}

(Received 5 August 1991; revised 8 September 1992)

\begin{abstract}
Coupled nonlinear partial differential equations, which describe a nonlinear resonant interaction between the fundamental and its first harmonic on a magnetohydrodynamic jet, are derived by the derivative expansion method. We investigate the spatial behaviour of the amplitude and phases. It is shown that the fluid surface is unstable in the neighbourhood of the first resonant wavenumber. In the steady state, it is observed that the general motion consists of both amplitude and phase modulated waves.
\end{abstract}

\section{Introduction}

The capillary instability of a jet has been a subject of considerable interest and has been investigated by a number of workers since the pioneering work of Lord Rayleigh [6]. The effect of nonlinear disturbances on the capillary instability of a hydrodynamic jet was examined by Yuen [8], Wang [7], Nayfeh [3], Nayfeh and Hassan [4] and Kakutani et al. [1]. It was shown by Kakutani et al. [1] that the complex amplitude of a quasimonochromatic travelling wave can be described by a nonlinear Schrödinger equation in a frame of reference moving with the group velocity. Lardner and Trehan [2] extended this result to include the effect of a uniform magnetic field. In fact they were the first to use the full hydromagnetic fluid equations and make no restriction to potential flows to study the modulational instability of a jet. They showed that the modulational instability can not be completely suppressed, and that the presence

\footnotetext{
${ }^{1}$ Centre for computer Science and Applications, Panjab University, Chandigarh - 160014, India.

${ }^{2}$ Dept of Chemical Engineering and Technology, Panjab University, Chandigarh - 160014, India.

(C) Australian Mathematical Society, 1994, Serial-fee code 0334-2700/94
} 
of a magnetic field does greatly increase the range of stable wave numbers. It was observed by them that for a hydromagnetic jet a significant feature of the analysis is the presence of a second harmonic resonance and the usual analysis is not valid in its neighbourhood. This type of resonance has been studied by various authors for capillary gravity waves on deep water, a self gravitating fluid cylinder and the hydromagnetic waves in a cold collisionless plasma. The slowly varying amplitudes of the two waves in resonance are described by a set of dynamical equations involving the fundamental and the first harmonic for a magnetohydrodynamic jet. It is shown that the jet is unstable in the presence of a magnetic field.

\section{Formulation of the problem}

We examine here a cylindrical fluid column of radius $R$ held together by surface tension; the fluid is assumed to be inviscid, incompressible and perfectly conducting, with a uniform magnetic field in the axial direction. The motion is assumed to be axially symmetric and the outer surface of the jet is distorted to $r=1+\eta(z, t)$, where $\eta(z, t)$ is the disturbance in the $r$-direction. In this discussion all lengths are measured in units of the radius $R$ of the cylinder and time is measured in units $R / A_{0}$, where $A_{0}$ is the Alfvén speed. The equations in the region $r \leq 1+\eta$ are:

$$
\begin{aligned}
\frac{\partial \mathbf{u}}{\partial t}+(\mathbf{u} \cdot \underset{\nabla}{\nabla}) \mathbf{u} & =-\underset{\sim}{\nabla} \Pi+(\mathbf{h} \cdot \underset{\sim}{\nabla}) \mathbf{h}, \\
\frac{\partial \mathbf{h}}{\partial t} & =(\mathbf{h} \cdot \underset{\sim}{\nabla}) \mathbf{h}-(\mathbf{u} \cdot \underset{\nabla}{\nabla}) \mathbf{h}, \\
\underset{\nabla}{\nabla} \mathbf{u}=0, \quad \underline{\nabla} \cdot \mathbf{h} & =0, \quad \Pi=\frac{p}{\rho}+.5 \mathbf{h}^{2},
\end{aligned}
$$

where $\mathbf{u},(4 \pi \rho)^{1 / 2} \mathbf{h}, p$ and $\rho$ represent the velocity field, the magnetic field, the pressure and the constant fluid density respectively. In the region $r \geq 1+\eta$, the magnetic field is expressible in terms of a potential $\phi$, that is,

$$
\mathbf{h}^{(0)}=A_{0} \mathbf{e}_{z}+\nabla \phi, \quad \nabla^{2} \phi=0 .
$$

The boundary conditions at $r=1+\eta$ are

$$
u_{r}=\frac{\partial \eta}{\partial t}+u_{z} \frac{\partial \eta}{\partial z}
$$




$$
\Pi=\frac{T}{\rho}\left((1+\eta)^{-1}\left\{1+\left(\frac{\partial \eta}{\partial z}\right)^{2}\right\}^{-1 / 2}-\frac{\partial^{2} \eta}{\partial z^{2}}\left\{1+\left(\frac{\partial \eta}{\partial z}\right)^{2}\right\}^{-3 / 2}\right)+\frac{1}{2} \mathbf{h}^{(0)^{2}}
$$

where $\mathrm{T}$ represents the fluid surface tension and $\mathbf{n}$ the unit normal to the surface. We choose units such that $T / \rho=1$. To determine an approximate solution to (1) - (3), we shall use the method of multiple time scales. If $\varepsilon$ is a small parameter measuring the size of perturbation, we introduce the space and time scales $z_{0}=z, z_{1}=\varepsilon z, t_{0}=t, t_{1}=\varepsilon t$. The partial derivatives are expressed in terms of slow scales according to

$$
\begin{aligned}
& \frac{\partial}{\partial z}=\frac{\partial}{\partial z_{0}}+\varepsilon \frac{\partial}{\partial z_{1}}, \\
& \frac{\partial}{\partial t}=\frac{\partial}{\partial t_{0}}+\varepsilon \frac{\partial}{\partial t_{1}}
\end{aligned}
$$

Moreover, the various physical variables are assumed to possess the following representations:

$$
f(r, z, t)=\sum_{n=0}^{2} \varepsilon^{n} f_{n}\left(r, z_{0}, z_{1}, t_{0}, t_{1}\right)+o\left(\varepsilon^{3}\right),
$$

where $f(r, z, t)$ is any of the variables $\mathbf{u}, \mathbf{h}, \Pi, \phi$ and $\eta$ with $\left(u_{0}, h_{0}, \Pi_{0}, \phi_{0}, \eta_{0}\right)=$ $\left(0, A_{0} \mathrm{e}_{z}, p_{0} / \rho_{0}+(1 / 2) A_{0}^{2}, 0,0\right)$. Substituting the expansions (4) - (5) into (1) (3), we obtain in the first and second orders respectively the following equations (in which we use the notation that $f_{, \alpha}=\partial f / \partial \alpha$ ).

a) First order equations:

$$
\begin{aligned}
\mathbf{u}_{1, t_{0}}+\nabla_{0} \Pi_{1}-A_{0} \mathbf{h}_{1, z_{0}} & =0, \\
\mathbf{h}_{1, t_{0}}-A_{0} \mathbf{u}_{1, z_{0}} & =0, \\
u_{1 r, r}+r^{-1} u_{1 r}+u_{1 z, z_{0}} & =0, \\
h_{1 r, r}+r^{-1} h_{1 r}+h_{12, z_{0}} & =0, \\
\nabla_{0}^{2} \phi_{1} & =0,
\end{aligned}
$$

and the boundary conditions on $r=1$ :

$$
\begin{aligned}
u_{1 r}-\eta_{1, t_{0}} & =0, \\
\Pi_{1}+\eta_{1, z_{0} z_{0}}-A_{0} h_{1 z}^{(0)} & =0, \\
h_{1 r}-h_{1 r}^{(0)} & =0,
\end{aligned}
$$


b) Second order equations :

$$
\begin{aligned}
& \mathbf{u}_{2, t_{0}}+\underset{\sim}{\nabla} \Pi_{2}-A_{0} \mathbf{h}_{2,2_{0}}=-\mathbf{u}_{1, t_{1}}-\Pi_{1, z_{1}} \mathbf{e}_{2}+A_{0} \mathbf{h}_{1, z_{1}}-\left(\mathbf{u}_{1} \cdot \underset{\sim}{\nabla}\right) \mathbf{u}_{1} \\
& +\left(\mathbf{h}_{1} \cdot \underset{\nabla_{0}}{ }\right) \mathbf{h}_{1} \text {, } \\
& \mathbf{h}_{2, t_{0}}-A_{0} \mathbf{u}_{2, z_{0}}=-\mathbf{h}_{1, t_{1}}+A_{0} \mathbf{u}_{1, z_{1}}+\left(\mathbf{h}_{1} \cdot{\underset{\sim}{0}}_{0}\right) \mathbf{u}_{1}-\left(\mathbf{u}_{1} \cdot{\underset{\sim}{0}}_{0}\right) \mathbf{h}_{1} \text {, } \\
& u_{2 r, r}+r^{-1} u_{2 r}+u_{2 z, 2_{0}}=-u_{1 z, z_{1}} \text {, } \\
& h_{2 r, r}+r^{-1} h_{2 r}+h_{2 z, 2_{0}}=-h_{1 z, z_{1}} \text {, } \\
& \nabla_{0}^{2} \phi_{2}=-2 \phi_{1,2021} \text {, }
\end{aligned}
$$

and the boundary conditions on $r=1$ :

$$
\begin{aligned}
& u_{2 r}-\eta_{2, t_{0}}= \eta_{1, t_{1}}+u_{1 z} \eta_{1, z_{0}}-\eta_{1} u_{1 r, r} \\
& \Pi_{2}+\eta_{2}+\eta_{2, z_{0} z_{0}}-A_{0} h_{2 z}^{(0)}=\eta_{1}^{2}- \frac{1}{2} \eta_{1, z_{0}}^{2}-2 \eta_{1, z_{0} z_{1}}-\eta_{1} \Pi_{1, r} \\
&+A_{0} \eta_{i} h_{1 z, r}^{(0)}+\frac{1}{2} \mathbf{h}_{1}^{(0)^{2}} \\
& h_{2 r}-h_{2 r}^{(0)}=-\eta_{1}\left(h_{1 r, r}-h_{1 r, r}^{(0)}\right)+\left(h_{1 z}-h_{1 z}^{(0)}\right) \eta_{1, z_{0}}
\end{aligned}
$$

where

$$
\nabla_{0}=\mathbf{e}_{r} \frac{\partial}{\partial r}+\mathbf{e}_{z} \frac{\partial}{\partial z_{0}}
$$

\section{Expansions}

The sinusoidal travelling wave solution of the first order problem governed by (6) - (7) can be written as

$$
\begin{aligned}
& {\left[u_{1 r}, u_{1 z}, \Pi_{1}, \eta_{1}\right]=\alpha\left[-i I_{1}(k r), I_{0}(k r), \frac{I_{0}(k r)}{\alpha}, \frac{I_{1}(k)}{\omega}\right] A\left(z_{1}, t_{1}\right) e^{i \psi}+\text { c.c., }} \\
& {\left[h_{1 r}, h_{1 z}, \phi_{1}\right]=\frac{k A_{0} \alpha}{\omega}\left[i I_{1}(k r),-I_{0}(k r),-\frac{i I_{1}(k) K_{0}(k r)}{k K_{1}(k)}\right] A\left(z_{1}, t_{1}\right) e^{i \psi}+c . c .,}
\end{aligned}
$$

where $\alpha=\omega k /\left(\omega^{2}-k^{2} A_{0}^{2}\right), \psi=k z_{0}-\omega t_{0}, c . c$. is the complex conjugate and the frequency $\omega$ satisfies the dispersion relation

$$
\omega^{2}=\frac{k\left(k^{2}-1\right)}{I_{a}}+\frac{k A_{0}^{2}}{I_{0}(k) K_{1}(k)}, \quad I_{a}=\frac{I_{0}(k)}{I_{1}(k)} .
$$

It is clear that (12) allows for an assigned value of $A_{0}$, a single positive root $\left(k_{c} \neq 0\right)$. We find that $\omega^{2}<0$ for $0<k<k_{c}$. The jet is, therefore, unstable for 


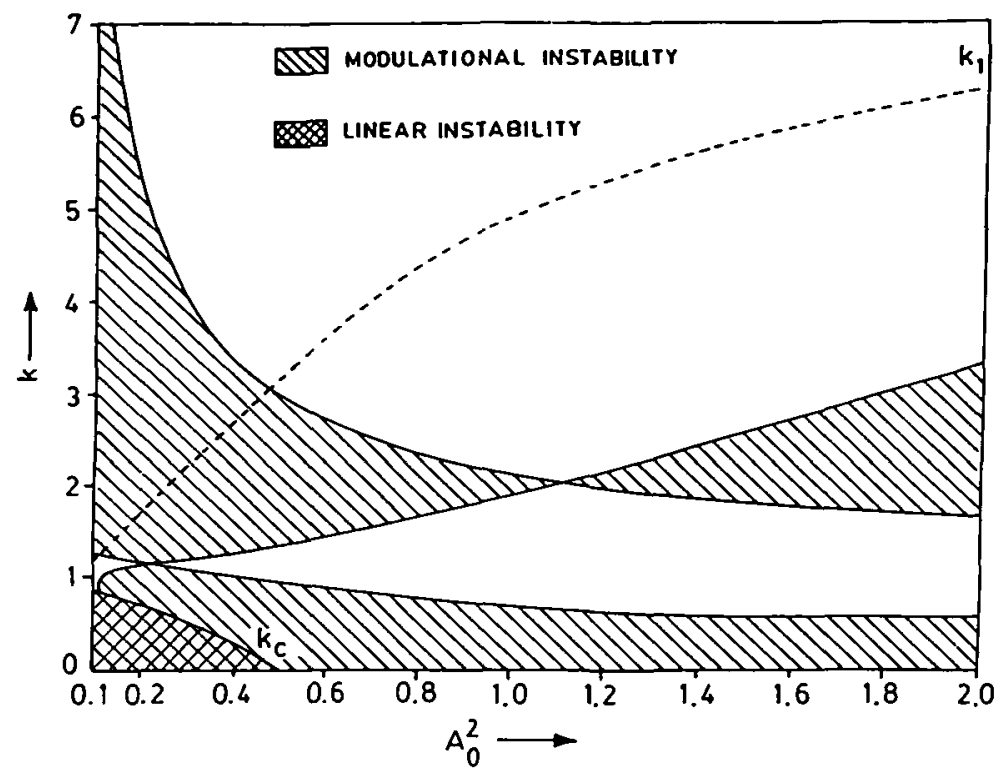

FIGURE 1. Instability regions in the $k-A_{0}^{2}$ plane. The dashed curve indicates the second harmonic resonance.

all deformations with wave numbers $k<k_{c}$, where $k_{c}$ depends on the strength of the magnetic field $A_{0}$. It is observed that the presence of a magnetic field $\left(A_{0} \neq 0\right)$ extends the region in which stable linear waves occur below $k_{c}=1$, and in fact $\omega^{2}>0$ for all wave numbers for $A_{0}^{2}>1 / 2$. In this paper, we assume that $\omega^{2}>0$ so that the first order solutions represent a uniformly travelling wave train.

We now investigate the conditions under which the two waves can interact resonantly. Harmonic resonance will occur for all wave numbers $k$ such that $(k, \omega)$ and $(n k, n \omega)$ for some integer greater than $n=1$ satisfy (12). The first resonant wave number $k_{1}$ corresponds to $n=2$ and is the solution of

$$
\frac{\left(4 k_{1}^{2}-1\right)}{I_{b}}-\frac{2\left(k_{1}^{2}-1\right)}{I_{a}}+A_{0}^{2}\left(\frac{1}{I_{0}\left(2 k_{1}\right) K_{1}\left(2 k_{1}\right)}-\frac{2}{I_{0}\left(k_{1}\right) K_{1}\left(k_{1}\right)}\right)=0,
$$

where

$$
I_{b}=I_{0}\left(2 k_{1}\right) / I_{1}\left(2 k_{1}\right) \text {. }
$$

It is found that for all $A_{0}^{2} \neq 0,(13 a)$ has roots, which leads to the presence of a second harmonic resonance. The variation of $k_{1}$ with $A_{0}^{2} \neq 0$ has been 
calculated and is shown in Figure 1. To describe the resonant interaction at or near $k_{1}$, we write

$$
\begin{aligned}
& {\left[u_{1 r}, u_{1 z}, \Pi_{1}, \eta_{1}\right]} \\
& \quad=\sum_{n=1}^{2} \alpha_{n}\left(-i I_{1}\left(k_{n} r\right), I_{0}\left(k_{n} r\right), \frac{I_{0}\left(k_{n} r\right)}{\alpha_{n}}, \frac{I_{1}\left(k_{n}\right)}{\omega_{n}}\right) A_{n} e^{i \psi_{n}}+c . c .,
\end{aligned}
$$

$\left[h_{1 r}, h_{1 z}, \phi_{1}\right]$

$$
=\sum_{n=1}^{2} \frac{k_{n} A_{0} \alpha_{n}}{\omega_{n}}\left(i I_{1}\left(k_{n} r\right),-I_{0}\left(k_{n} r\right),-\frac{i I_{1}\left(k_{n}\right) K_{0}\left(k_{n} r\right)}{k_{n} K_{1}\left(k_{n}\right)}\right) A_{n} e^{i \psi_{n}}+c . c .,
$$

where

$$
\begin{aligned}
& \omega_{n}^{2}=\frac{k_{n}}{I_{0}\left(k_{n}\right)}\left(\left(k_{n}^{2}-1\right) I_{1}\left(k_{n}\right)+\frac{A_{0}^{2}}{K_{1}\left(k_{n}\right)}\right), \\
& \psi_{n}=k_{n} z_{0}-\omega_{n} t_{0}, \\
& \alpha_{n}=\frac{\omega_{n} k_{n}}{\omega_{n}^{2}-k_{n}^{2} A_{0}^{2}}, \quad k_{2}=2 k_{1}, \quad \omega_{2}=2 \omega_{1} .
\end{aligned}
$$

On substituting the first order solutions into the second order problem governed by (8) - (9), we obtain for the second order equations:

$$
\begin{aligned}
& u_{2 r, t_{0}}+\Pi_{2, r}^{\prime}-A_{0} h_{2 r, z_{0}}= i \sum_{n=1}^{2}\left(\alpha_{n} I_{1}\left(k_{n} r\right)\left(A_{n, t_{1}}+M_{n} A_{n, z_{1}}\right) e^{i \psi_{n}}\right)+c . c ., \quad(16 \mathrm{a}) \\
& u_{2 z, t_{0}}+\Pi_{2, z_{0}}^{\prime}-A_{0} h_{2 z, z_{0}}=-i \sum_{n=1}^{2}\left(\left\{\alpha_{n} I_{0}\left(k_{n} r\right)\left(A_{n, t_{1}}+M_{n} A_{n, z_{1}}\right)\right.\right. \\
& h_{2 r, t_{0}}-A_{0} u_{2 r, z_{0}}=\left.\left.-i A_{0}\left(k_{n} r\right) A_{n, z_{1}}\right\} e^{i \psi_{n}}\right)+c . c ., \\
& h_{2 z, t_{0}}-A_{0} u_{2 z, z_{0}}=A_{0} \sum_{n=1}^{2}\left(R_{n} I_{1}\left(k_{n} r\right)\left(A_{n, t_{1}}+W_{n} A_{n, z_{1}}\right) e^{i \psi_{n}}\right)+c . c ., \\
&\left.\left.u_{2 r, r}+k_{n} r\right)\left(A_{n, t_{1}}+W_{n} A_{n, z_{1}}\right) e^{i \psi_{n}}\right)+c . c .,(16 \mathrm{~d}) \\
& u_{2 r}+u_{2 z, z_{0}}=-\sum_{n=1}^{2}\left(\alpha_{n} I_{0}\left(k_{n} r\right) A_{n, z_{1}} e^{i \psi_{n}}\right)+c . c ., \\
& h_{2 r, r}+r^{-1} h_{2 r}+h_{2 z, z_{0}}=A_{0} \sum_{n=1}^{2}\left(R_{n} I_{0}\left(k_{n} r\right) A_{n, z_{1}} e^{i \psi_{n}}\right)+c . c .,
\end{aligned}
$$




$$
\phi_{2 r, r}+r^{-1} \phi_{2 r}+\phi_{2 z, z_{0}}=-2 A_{0} \sum_{n=1}^{2}\left(\frac{R_{n} I_{1}\left(k_{n}\right)}{K_{1}\left(k_{n}\right)} K_{0}\left(k_{n} r\right) A_{n, z_{1}} e^{i \psi_{n}}\right)+c . c .,
$$

where

$$
\begin{aligned}
& \Pi_{2}^{\prime}=\Pi_{2}+\frac{1}{2} R_{1}\left(I_{0}^{2}\left(k_{1} r\right)-I_{1}^{2}\left(k_{1} r\right)\right) A_{1}^{2} e^{2 i \psi_{1}} \\
& +R_{1}\left(I_{0}\left(k_{1} r\right) I_{0}\left(k_{2} r\right)+I_{1}\left(k_{1} r\right) I_{1}\left(k_{2} r\right)\right) A_{2} \bar{A}_{1} e^{i \psi_{1}}, \\
& M_{n}=\frac{A_{0}^{2}}{W_{n}}, \quad R_{n}=\frac{\alpha_{n}}{W_{N}}, \quad W_{n}=\frac{\omega_{n}}{k_{n}} .
\end{aligned}
$$

The boundary conditions reduced to $r=1$ are:

$$
\begin{aligned}
& u_{2 r}-\eta_{2, t_{0}} \\
& =\sum_{n=1}^{2}\left(\frac{\alpha_{n} I_{1}\left(k_{n}\right)}{\omega_{n}} A_{n, t_{1}} e^{i \psi_{n}}\right)+\frac{i \alpha_{1}^{2}}{\omega_{1}}\left(2 k_{1} I_{0}\left(k_{1}\right) I_{1}\left(k_{1}\right)-I_{1}^{2}\left(k_{1}\right)\right) A_{1}^{2} e^{2 i \psi_{1}} \\
& +\frac{i \alpha_{1} \alpha_{2}}{2 \omega_{1}}\left(k_{1}\left\{I_{1}\left(k_{2}\right) I_{0}\left(k_{1}\right)+2 I_{1}\left(k_{1}\right) I_{0}\left(k_{2}\right)\right\}-I_{1}\left(k_{1}\right) I_{1}\left(k_{2}\right)\right) A_{2} \bar{A}_{1} e^{i \psi_{1}} \\
& +c . c \text {., } \\
& \Pi_{2}+\eta_{2}+\eta_{2, z_{0} z_{0}}-A_{0} h_{2 z}^{(0)} \\
& =-2 i \sum_{n=1}^{2}\left(R_{n} I_{1}\left(k_{n}\right) A_{n, z_{1}} e^{i \psi_{n}}\right) \\
& +\left(\frac{\alpha_{1} \alpha_{2}}{\omega_{1}^{2}}\left\{1-k_{1}^{2}-.5 k_{1}^{2} A_{0}^{2}\left(1-4 K_{a 1} K_{a 2}\right)-2.5 \frac{k_{1}^{2}}{R_{1}}\right\}\right) \\
& \times I_{1}\left(k_{1}\right) I_{1}\left(k_{2}\right) A_{2} \bar{A}_{1} e^{i \psi_{1}} \\
& +\left(\frac{\alpha_{1}^{2} I_{1}^{2}\left(k_{1}\right)}{\omega_{1}^{2}}\left\{1+.5 k_{1}^{2}-\omega_{1}^{2}+.5 k_{1}^{2} A_{0}^{2}\left(K_{a 1}^{2}-1\right)\right\}\right) A_{1}^{2} e^{2 i \psi_{1}} \\
& +c . c \text {., } \\
& =i \sum_{n=1}^{2}\left(R_{n} A_{0} I_{1}\left(k_{n}\right) K_{a n} e^{i \psi_{n}}\right)-\frac{2 i \alpha_{1}^{2} k_{1}^{2} A_{0} I_{1}^{2}\left(k_{1}\right)}{\omega_{1}^{2}}\left(K_{a 1}-I_{a 1}\right) A_{1}^{2} e^{2 i \psi_{1}} \\
& -.5 i R_{1}^{2} A_{0}\left(K_{a 1}+K_{a 2}+I_{a 1}+2 I_{a 2}\right) A_{2} \bar{A}_{1} e^{i \psi_{1}}+c . c .,
\end{aligned}
$$

where

$$
K_{a n}=K_{0}\left(k_{n}\right) / K_{1}\left(k_{n}\right), \quad I_{a n}=I_{0}\left(k_{n}\right) / I_{1}\left(k_{n}\right)
$$


After some straight forward reductions we obtain the uniformly valid solution of the second order problem as:

$$
\begin{aligned}
\eta_{2}=-i \sum_{n=1}^{2}\left(\frac{\alpha_{n}}{\omega_{n}^{2}}\left\{I_{1}\left(k_{n}\right) A_{n, t_{1}}+\omega_{n} I_{2}\left(k_{n}\right) A_{n, z_{1}}\right\} e^{i \psi_{n}}\right) & \\
& +\frac{\alpha_{1}^{2}}{2 \omega_{1}^{2}} I_{1}^{2}\left(k_{1}\right)\left(2 k_{1} I_{a 1}-1\right) A_{1}^{2} e^{2 i \psi_{1}} \\
& +\frac{\alpha_{1} \alpha_{2}}{2 \omega_{1}^{2}}\left(k_{1} I_{1}\left(k_{1}\right) I_{0}\left(k_{2}\right)\left\{2+\frac{I_{a 1}}{I_{a 2}}\right\}-I_{1}\left(k_{2}\right) I_{1}\left(k_{1}\right)\right) A_{2} \bar{A}_{1} e^{i \psi_{1}} \\
& +c . c .,
\end{aligned}
$$

Equations (19) together with (18b) - (18c) lead to the dynamical equations for the coupled amplitude as

$$
\begin{aligned}
& \frac{\partial A_{1}}{\partial t_{1}}+U_{1} \frac{\partial A_{1}}{\partial z_{1}}=i q_{1} A_{2} \bar{A}_{1} e^{i \tau}, \\
& \frac{\partial A_{2}}{\partial t_{1}}+U_{2} \frac{\partial A_{2}}{\partial z_{1}}=i q_{2} A_{1}^{2} e^{i \tau},
\end{aligned}
$$


where

$$
\begin{aligned}
U_{i}=\frac{d \omega_{i}}{d k}, & i=1,2, \\
q_{1}=-\frac{k_{1}}{2 \alpha_{1} I_{0}\left(k_{1}\right)}\left(I_{1}\left(k_{1}\right) I_{1}\left(k_{2}\right) \frac{\alpha_{1} \alpha_{2}}{\omega_{1}^{2}}\right. & \times\left[1-k_{1}^{2}\left\{1-.5 A_{0}^{2}\left(1+4 K_{a 1} K_{a 2}\right)\right\}-2.5 R_{1} I_{1}\left(k_{1}\right) I_{1}\left(k_{2}\right)\right] \\
& \times\left(1-k_{1}^{2}\right)\left(\frac{\alpha_{1} R_{1}}{2 \omega_{1}} I_{1}\left(k_{1}\right) I_{1}\left(k_{2}\right)\left\{2 I_{a 2}+\frac{I_{a 2} I_{1}\left(k_{2}\right)}{I_{2}\left(k_{1}\right)}-\frac{1}{k_{1}}\right\}\right) \\
& +\frac{\alpha_{1} R_{1}}{2 \omega_{1}} A_{0}^{2} K_{a 1}\left\{\frac{I_{1}\left(k_{1}\right)}{K_{1}\left(k_{2}\right)}+\frac{I_{1}\left(k_{2}\right)}{K_{1}\left(k_{1}\right)}\right\} \\
& \left.+R_{1} I_{1}\left(k_{1}\right) I_{1}\left(k_{2}\right)\left\{1+I_{a 1}+I_{a 2}\right\}\right), \\
& \frac{k_{2}}{2 \alpha_{2} I_{0}\left(k_{2}\right)}\left[.5 R_{1}\left(I_{0}^{2}\left(k_{1}\right)-I_{1}^{2}\left(k_{1}\right)\right)\right. \\
& +\frac{\alpha_{1}^{2}}{\omega_{1}^{2}} I_{1}^{2}\left(k_{1}\right)\left\{1+.5 k_{1}^{2}-\omega_{1}^{2}+.5 k_{1}^{2} A_{0}^{2}\left(K_{a 1}^{2}-1\right)\right\} \\
& -\frac{\alpha_{1}^{2}}{2 \omega_{1}^{2}}\left(1-k_{2}^{2}\right)\left\{2 k_{1} I_{0}\left(k_{1}\right) I_{1}\left(k_{1}\right)-I_{1}^{2}\left(k_{1}\right)\right\} \\
& \left.+4 \alpha_{1} R_{2} A_{0}^{2} K_{a 2} \frac{I_{1}\left(k_{2}\right)}{\omega_{2} K_{1}\left(k_{1}\right)}\right],
\end{aligned}
$$

and the detuning parameter

$$
\tau=\frac{\left(k_{2}-2 k_{1}\right) x_{1}}{\varepsilon}-\frac{\left(\omega_{2}-2 \omega_{1}\right) t_{1}}{\varepsilon} .
$$

We have calculated the values of $q_{1}$ and $q_{2}$ for various values of $A_{0}^{2} \neq 0$ and it has been observed that $q_{1}$ and $q_{2}$ have opposite signs. If we let $A_{m}=\left(a_{m} / 2\right) \exp \left(i \theta_{m}\right)$ with $a_{m}$ and $\theta_{m}$ real and slowly varying functions of the slower variables $z_{1}$ and $t_{1}$ in (20) - (21), we get

$$
\begin{aligned}
& \frac{\partial a_{1}}{\partial t_{1}}+U_{1} \frac{\partial a_{1}}{\partial z_{1}}=-q_{1} \frac{a_{1} a_{2}}{2} \sin (\alpha), \\
& \frac{\partial a_{2}}{\partial t_{1}}+U_{2} \frac{\partial a_{2}}{\partial z_{1}}=q_{2} \frac{a_{1}^{2}}{2} \sin (\alpha), \\
& \frac{\partial \theta_{1}}{\partial t_{1}}+U_{1} \frac{\partial \theta_{1}}{\partial z_{1}}=\frac{q_{1} a_{2}}{2} \cos (\alpha),
\end{aligned}
$$




$$
\frac{\partial \theta_{2}}{\partial t_{1}}+U_{2} \frac{\partial \theta_{2}}{\partial z_{1}}=\frac{q_{1} a_{1}^{2}}{2 a_{2}} \cos (\alpha)
$$

where

$$
\alpha=\theta_{2}-2 \theta_{1}+\tau .
$$

It appears difficult to obtain the solutions of (26) - (29) subject to general initial conditions; we investigate the spatial variations of amplitude and phases in the $\varepsilon$ neighbourhood of the first resonant wave number, i.e., $\omega_{2}=2 \omega_{1}$, $k_{2}=2 k_{1}+0(\varepsilon)$. In this case, we get after some reductions:

$$
\begin{aligned}
a_{1}^{2}+v a_{2}^{2} & =E \\
\left(d \chi / d z_{1}\right)^{2} & =G(\chi),
\end{aligned}
$$

where

$$
\begin{aligned}
v & =U_{2} q_{1} /\left(U_{1} q_{2}\right), \\
G(\chi) & =\frac{E q_{2}^{2}}{U_{2}^{2}}\left(\chi(1-v \chi)^{2}-\frac{1}{E}\left[\frac{L}{E}-\frac{\sigma U_{2}}{q_{2}} \chi\right]^{2}\right), \\
\chi & =a_{2}^{2} / E, \quad \sigma=\left(k_{2}-2 k_{1}\right) / \varepsilon .
\end{aligned}
$$

Here $\mathrm{E}$ and $\mathrm{L}$ are the constants of integration. The stability of the fluid surface is dependent upon the roots of the algebric equation $G(\chi)=0$ (see Nayfeh and Mook [5]). For the particular initial conditions $a_{1}^{2}=E, a_{2}=0$ at $z=z_{1}=0$, we get $L=0$ and

$$
\left(d \chi / d z_{1}\right)^{2}=R \chi\left((1-v \chi)^{2}-2 \Omega \chi\right),
$$

where $R=E q_{2}^{2} / U_{2}^{2}$ and $\Omega=\sigma^{2} U_{2}^{2} /\left(2 E q_{2}^{2}\right)$. Therefore, since $\nu<0$, the motion is stable only if $\Omega>2|v|$. The fluid surface is unstable and the displacement grows if $v<0$ and the detuning parameter $\sigma$ is small enough so that $\sigma^{2}<4|v| E q_{2}^{2} / U_{2}^{2}$. In particular, at perfect resonance, (i.e., $\sigma=0$ ) and $\nu<0$, the fluid surface is always unstable. Thus at the second harmonic resonance the fluid motion becomes unbounded and hence results in an instability. Lardner and Trehan [2] calculated the modulationally stable and unstable regions which are shown in Figure 1. We observe that the first resonant wave number where the solutions of Lardner and Trehan [2] are not valid lies in the modulationally stable region for some values of $A_{0}^{2} \neq 0$. We have shown that in that region, at perfect resonance, the fluid motion results in an instability at the second harmonic resonance. 


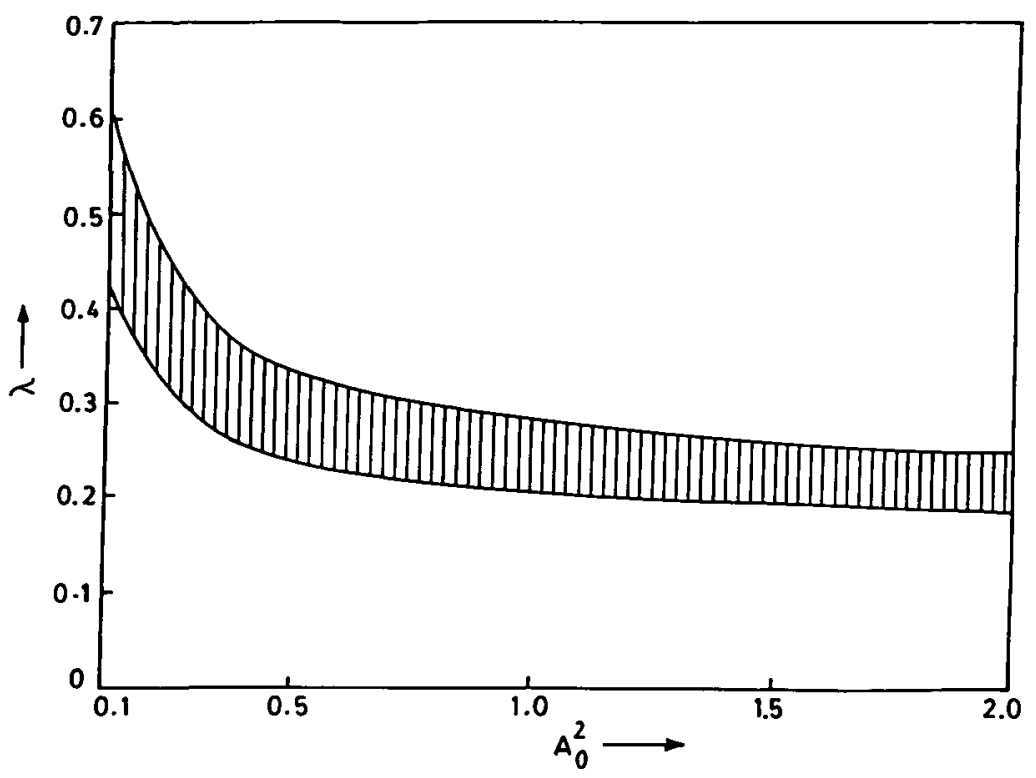

Figure 2. $\lambda$ versus $A_{0}^{2}$ for $\beta_{2} \beta_{1}^{-1}>0$.

We now examine the steady state solutions of (20) - (21) and assume $A_{m}=$ $f_{m}(\xi) \exp \left(i \mu m z_{1}\right), f_{m}(\xi)=a_{m} \exp (i \Phi m)$, with $m=1,2$ and $\xi=t_{1}-\lambda z_{1}$, where $\mu$ and $\lambda$ are real constants. Also $a_{m}$ and $\Phi_{m}$ are parameters of slow scale $z_{1}$ and $t_{1}$. Equations (20) and (21) with $\tau=0$ leads to

$$
\begin{gathered}
\beta_{1} a_{1}^{2}+\beta_{2} a_{2}^{2}=E_{0}, \\
a_{1}^{\prime 2}+\frac{\lambda_{0}^{2}}{a_{1}^{2}}+a_{1}^{2}\left(\frac{\delta^{2}}{\beta_{1}^{2}}-\frac{E_{0}}{\beta_{1}^{2} \beta_{2}}\right)+\frac{a_{1}^{4}}{\beta_{1} \beta_{2}}+\frac{2 \lambda_{0} \delta}{\beta_{1}}=0
\end{gathered}
$$

where $\beta_{m}=\left(1-\lambda U_{m}\right) / q_{m}, m=1,2$ and $\delta=\mu \beta_{1}\left[U_{1} /\left(q_{1} \beta_{1}\right)-U_{2} /\left(q_{2} \beta_{2}\right)\right]$. $E_{0}$ and $\lambda_{0}$ are the constants of integration. In view of the apparent singularity in (38), where $a_{1} \longrightarrow o, \lambda_{0}=0$, it is convenient to express this result in terms of energy $E_{1}$ of the $A_{1}$ oscillator to $\mathrm{O}(1)$, with $E_{1}=a_{1}^{2} / 2$. Thus (38) reduces to

$$
E_{1}^{2}+\lambda_{0}^{2}-\frac{8 E_{1}^{2}}{\beta_{1} \beta_{2}}\left(\frac{E_{0}}{2 \beta_{1}}-E_{1}\right)+\frac{4 \delta E_{1}}{\beta_{1}}\left(\lambda_{0}+\frac{\delta E_{1}}{\beta_{1}}\right)=0
$$

The solution of (39) exists only if $E_{1}^{2}>0$ and is consistent with other integrals of motion for values of $\lambda_{0}$ such that $\beta_{2} \beta_{1}^{-1}>0$ and $0<\lambda_{0}^{2}<4 E_{0}^{3} /\left(27 \beta_{1}^{4} \beta_{2}\right)$. 
The values of $\lambda$ for which $\beta_{2} \beta_{1}^{-1}>0$ are calculated for various values of $A_{0}^{2} \neq 0$ and shown in Figure 2. The motion is bounded when $\lambda$ lies within the shaded region. These bounded solutions consist of both amplitude and phase modulated waves (see Nayfeh and Mook [5]).

\section{Acknowledgements}

The authors would like to express their gratitude to Prof. S.K. Trehan for a number of valuable suggestions. We are also indebted to the referee for his comments which helped to improve the original manuscript.

\section{References}

[1] T. Kakutani, Y. Inoue and T. Kan, "Nonlinear capillary waves on the surface of liquid column”, J. Phys. Soc. Japan 37 (1974) 529-538.

[2] R.W. Lardner and S.K.Trehan, "Modulational instability of a magnetohydrodynamic jet", Astrophys. Space Sci. 96 (1983) 261-281.

[3] A.H. Nayfeh, "Nonlinear stability of a liquid jet", Phys. of Fluids 13 (1970) 841-847.

[4] A.H. Nayfeh and S.D. Hassan, "The method of multiple scales and nonlinear dispersive waves", J. Fluid Mech. 48 (1971) 463-475.

[5] A.H. Nayfeh and D. Mook, Nonlinear Oscillations (Interscience, 1979).

[6] Lord Rayleigh, The theory of sound, Volume II (Dover Publications, Inc., New York, 1945).

[7] D.P. Wang, "Finite amplitude effect on the stability of a jet of circular cross section", J. Fluid Mech. 34 (1968) 299-313.

[8] M.C. Yuen, "Nonlinear capillary instability of a liquid jet”, J. Fluid Mech. 33 (1968) 151-163. 